\title{
INFLUENCE OF PARTICLES ON PARTIAL DISCHARGES AND BREAKDOWN IN OIL
}

\author{
K.N. Mathes and J.M. Atkins \\ General Electric Corporate Research and Development \\ Schenectady, N.Y.
}

\begin{abstract}
Particles influence both $A C$ and DC voltage breakdown in insulating oil. Larger and conducting particles decrease the breakdown voltage most. For both DC and AC the ratio, of breakdown voltage for clean oil to oil with non-conducting particles is nearly the same. For conducting particles the ratio for DC is generally larger than for AC.

Particle concentration and motion is influenced by DC and AC stress in different ways. Particles may be drawn into or expelled from high stress regions. Fine particles may agglomerate or be dispersed. Particle chains may develop. These complex physical factors have not been related directly to voltage breakdown in the oil Particles may cause partial discharges to develop in oil but these discharges also have not been related directly to short time breakdown of the oil.
\end{abstract}

\section{Introduction}

The influence of particles on the voltage breakdown in insulating oil and other liquids has been recognized for many years. Hirobe1 noted the influence of moist cellulose fibers 60 years ago. The adverse effect of moisture and other contaminants (such as acidic products from oxidation) in combination with particles has also been recognized ${ }^{1}$. $\mathrm{Kok}^{2}$ has emphasized the importance of particles in breakdown in insulating oil. He considers the electrophoretic motion of charged particles, the development of particle chains or bridges, field enhancement by particles, corona discharges, and other aspects. In general, Kok considered very small particles, $50 \AA$ to $200 \AA$, but as noted as well the possibility of flocculation (aggregation) to give particles in the range of $\sim 10^{3}$ $\AA$ to $10^{5} \AA(1$ to $100 \mu \mathrm{m})$. He considered the oscillation of particles in AC fields and has calculated the particle size in a given liquid and electric field so that the particle might completely traverse the gap between electrodes.

However, for the last 25 years, most research on voltage breakdown in liquids has been directed primarily toward obtaining the highest "intrinsic" value. For this purpose, uniform fields, small gaps, and very pure particle-free liquids were used. Even so, despite stringent efforts to remove them by very careful filtration, particles still "plagued" many research workers as reported by Gallagher $^{3}$ Goldschwartz and Blaise ${ }^{4}$ have filtered superfluid helium through a "superleak" Vyccafilter to remove particles down to $\sim 40 \AA$, and found thereby continuing increases in electric strength. Krasucki ${ }^{5}$ has proposed that submicroscopic particles are inherently important in an alternative version of the bubble mechanism of intrinsic breakdown to that proposed by Sharbaugh and Watson ${ }^{6}$ Dakin and Hughes ${ }^{7}$ report micro-discharges which they attribute to the charge transfer taking place as a charged particle contacts the electrode to which it is attracted. Some differences and disagreements exist, and the work of a number of authors are summarized by Gallagher? 
Crawley and Angerer8 have reported DC breakdown in oil that had been filtered through various filters with porosites ranging from a 0.05 to $1.5 \mu \mathrm{m}$. Filtering increased the breakdown strength. However, with an increase in gap distance between the electrodes, the breakdown stress decreased for the oil with the smallest particles and increased for the larger particles. This result suggests that in the longer gaps the larger particles might be removed from the oil under the influence of the electric field or that different breakdown mechanisms are involved.

In this work the influence of various kinds of particles in oil on both the AC and DC voltage breakdown has been studied. Partial discharges, visual performance and particle count have also been recorded where feasible. Emphasis has been placed in practical application rather than theoretical aspects.

\section{Particle Characterization}

Many techniques have been used for counting and determining the size and shape of particles suspended in liquid. The subject is so complex that space does not permit a detailed discussion here. In brief, small particles have been shown to form larger particles under some conditions. In other situations the aggregates may be dispersed to form smaller particles again.

Light and scanning electron microscopy (SEM) have been very useful in showing the shape, size and aggregation of particles in oil but particle count is difficult. Particle count in transparent liquids can be achieved by rather complex instrumentation using right angle or forward (Rayleigh) scattering of a light beam. By accepting certain compromises, commercial instruments have become available using these light scattering techniques. For much of this work a Royco counter has been used to obtain particle count in 5 preselected size ranges from 2 to $300 \mu \mathrm{m}$. With this instrument the particles are counted as $100 \mathrm{ml}$ of oil is passed through a glass capillary. The Royco instrument counts aggregates as large particles and the possibility also exists that the capillary itself may influence the degree of aggregation or dispersion.

A Model ILI 1000 Spectrex counter has been used to make particle count measurements in liquid held in transparent containers up to a maximum of $200 \mathrm{~mm}$ in depth. The Spectrex counter uses a helium-neon laser beam which is focused to a small, defined volume $(10 \mathrm{ml})$ within the liquid. Total particle count over a predetermined range between 2 and $100 \mu \mathrm{m}$ can be determined in less than a minute. The Spectrex instrument also counts aggregates as large particles. With care reasonably good agreement has been obtained between the Royco and Spectrex counters. The relatively large liquid volume with which the Spectrex counter can be used has made it possible to determine the influence of voltage stress and other factors on the particle count

\section{Particle Count and Motion with Voltage Stress}

The Spectrex counter permitted for the first time a particle count in oil while voltage stress was simultaneously applied. To stay within the liquid depth limit of the counter, special test cells were built In one cell a $1 / 2$ " dia. hemisphere was spaced from another such electrode in horizontal alignment The spacing was at least $2 \mathrm{~mm}$ to permit the laser beam to be focused directly between the electrodes.

In Fig. 1 (the electrode geometry is indicated in the figure) particle count was measured without voltage and after one minute intervals as the DC voltage was increased in $5 \mathrm{kV}$ steps to $25 \mathrm{kV}$. The voltage was then decreased similarly in $5 \mathrm{kv}$ steps. No particles were purposely added to the "clean" oil but particle motion between the electrodes could be clearly seen in the red laser beam of the Spectrex counter (The oil was not stirred as it is in the ASTM D1816 test so that particle motion induced by voltage stress could be observed). The particle count increased as voltage was increased. The particle motion back and forth between the electrodes also could be observed to increase with voltage. However, as the voltage was decreased, the particles 
appeared to stay suspended for some time. In Fig. 1 the voltage was raised as fast as the counting time would permit In other tests the DC voltage was raised much more slowly without greatly influencing the results. However, with $A C$ voltage the time of voltage application at a fixed voltage does influence the particle count as shown in Fig. 2. The oscillatory particle motion observed in the laser beam differs markedly from the back and forth motion with DC.

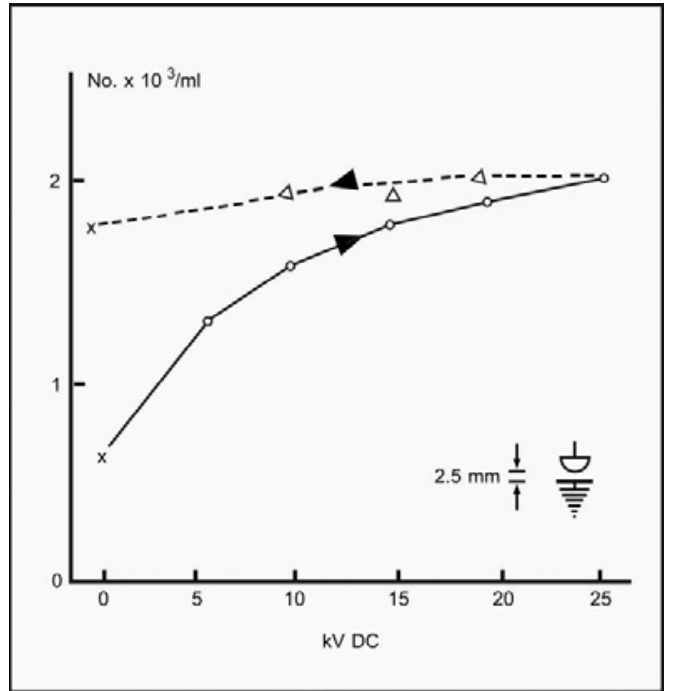

Fig. 1: In situ particle count vs positive DC voltage in clean oil; $2.5 \mathrm{~mm}$-diameter hemisphere spaced 2.5 $\mathrm{mm}$ from a plane (vertical).

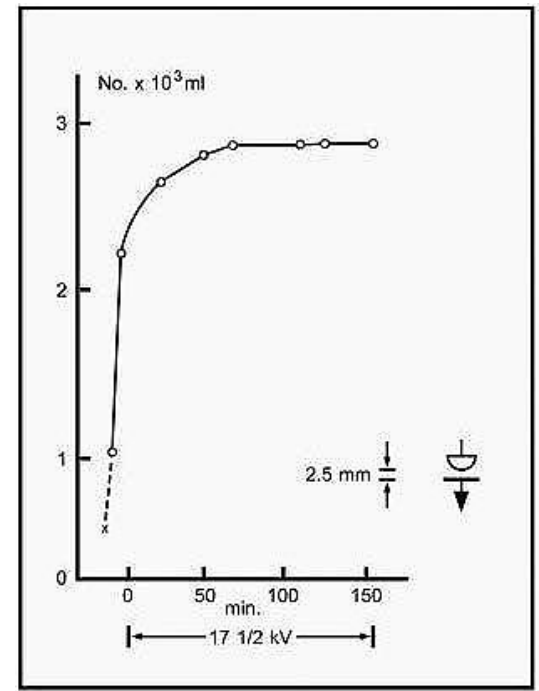

Fig. 2: In situ pulse count vs time at $171 / 2 \mathrm{kV}, 60 \mathrm{~Hz}$ voltage; $2.5 \mathrm{~mm}$-diameter hemisphere to plane spaced; $2.5 \mathrm{~mm}$ (vertical). Clean line oil (x-initial count at zero volts).

The visual observations in the laser beam were as instructive as the particle count. Particle chains were not observed with $A C$ voltage even when particles were purposely added or were produced by voltage breakdowns. With DC, the carbonaceous particles after a breakdown sometimes did line up in a "chain" so as to almost completely bridge the gap. However, as the DC voltage approached breakdown the particle "bridge" between the electrodes became unstable and broke up before voltage breakdown occurred.

Very fine metal particles tend to drop out of oil but may be kept suspended by voltage stress as shown in Fig. 3 where the horizontal electrode alignment might be expected to promote fall Out. In this case a small amount of fine iron powder was added to the oil. The initial count (shown by Fig. 3) at zero voltage is about the same as for a clean oil and at $5 \mathrm{kV}$ the iron particles did not move into the gap. At 10 and $14 \mathrm{kV}$ DC the iron particles could be seen to move into the gap and the particle count increased. As the voltage was held at $14 \mathrm{kV}$ the particle count first increased and then decreased over 30 minutes. The visual observations tended to confirm that agglomeration to larger particles (and decreased count) took place with time. When the voltage was reduced to zero the iron particles fell out quickly and the particle count decreased by a factor of almost ten over that for the initial measurement in clean Oil It seems likely that in the process of agglomeration some of the original particles are picked up and later carried out of the oil. 


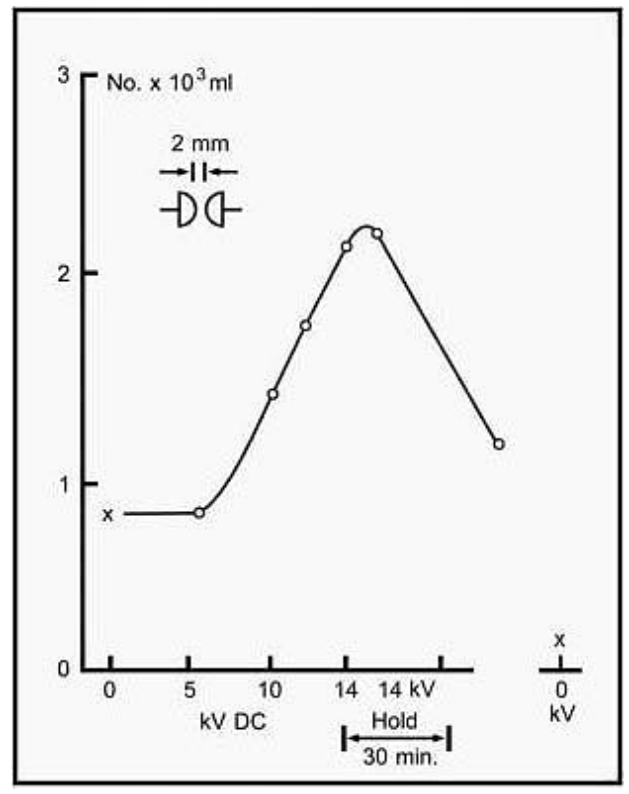

Fig. 3: In situ particle count vs DC voltage; $2.5 \mathrm{~mm}$ diameter hemispheres spaced $2 \mathrm{~mm}$ (horizontal).

Iron (carbonyl) powder in clean line Oil

Both still and moving pictures of suspended iron particles under DC and AC voltage stress have been made. Others, including Darveniza ${ }^{10}$ have also studied particle motion with similar results. For our studies a curved upper electrode to plane configuration has been used. In some cases the curved upper electrode has been replaced with a $12.5 \mathrm{~mm}$ steel tube electrode to produce a very non-uniform field. The oil can be degassed and the cell can be made corona free up to about $90 \mathrm{kV}$. Added particles are simply placed on the bottom $100 \mathrm{~mm}$ dia disc electrode. In this case a pressboard barrier around the bottom electrode prevents the particles from becoming trapped in the bottom of the cell. Electrode spacings up to $25 \mathrm{~mm}$ have been used.

Since partial discharges will be discussed next, it is worthwhile to report the visible observations made in total darkness. With the steel ball, visual tiny sparks or arcs are sometimes but not always easily seen as the ball approaches the electrodes. With the fine iron particles, such tiny arcs have never been observed visually. At voltages just below breakdown, a faint "glow" has been observed at the edge of the tube electrode in the presence of fine iron particles. Probably the discharges are of such small magnitude that they cannot be seen as discrete entities by the naked eye.

\section{Partial Discharges}

It has been possible to combine Spectrex particle count and partial discharge measurements, as illustrated by the results in Fig. 6. For this purpose, a needle to plane gap was used with a $25 \mathrm{~mm}$ spacing which could be made corona free up to about 50kV and still meet the size limitation for particle counting. For this geometry, no increase in particle count and no corona was observed with AC stress before breakdown. With a positive DC voltage on the needle, particle concentration started to increase at $10 \mathrm{kV}$ but no partial discharges (above $2 \mathrm{pC}$ ) were observed below $35 \mathrm{kV}$. 


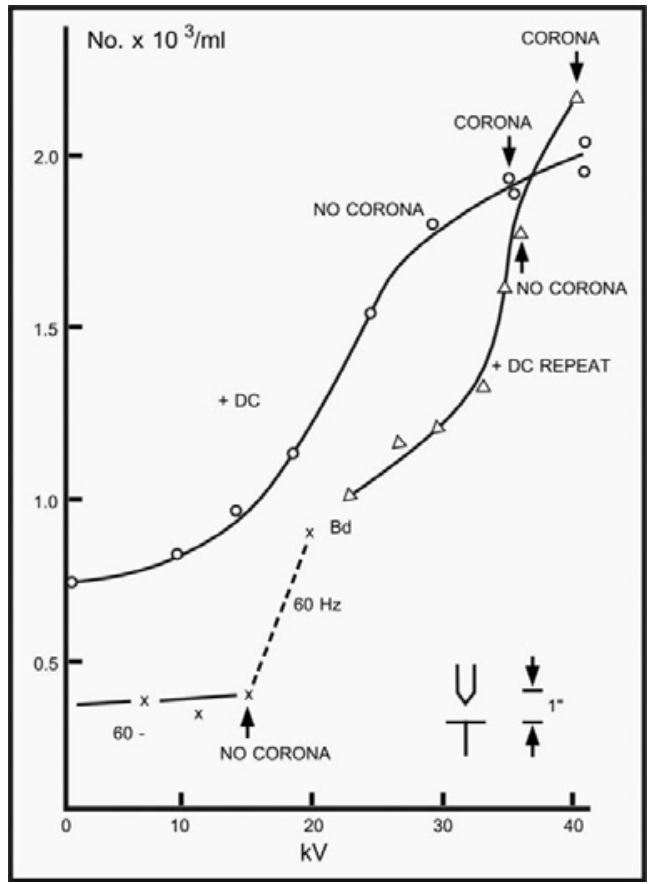

Fig. 6: In situ particle count vs $60 \mathrm{~Hz}$ and positive DC voltage. Clean line oil with added iron (carbonyl) particles. Needle-to-plane spaced 25 $\mathrm{mm}$ (vertical).

With the larger test cell, particle count could not be made in situ. The pulse repetition rate for a tube electrode in the larger cell is shown in Fig. 7. With this geometry and added iron particles, discharges are obtained with AC voltage before breakdown. The performance with DC stress of both polarities is also shown. Some apparent but minor inconsistencies between these results and those in Fig. 6 are believed to be related to the larger dimension of the sharp tube electrode which gives many more sites on which discharges can occur. With both geometries, no marked increase in discharge repetition rate magnitude was observed just before breakdown.

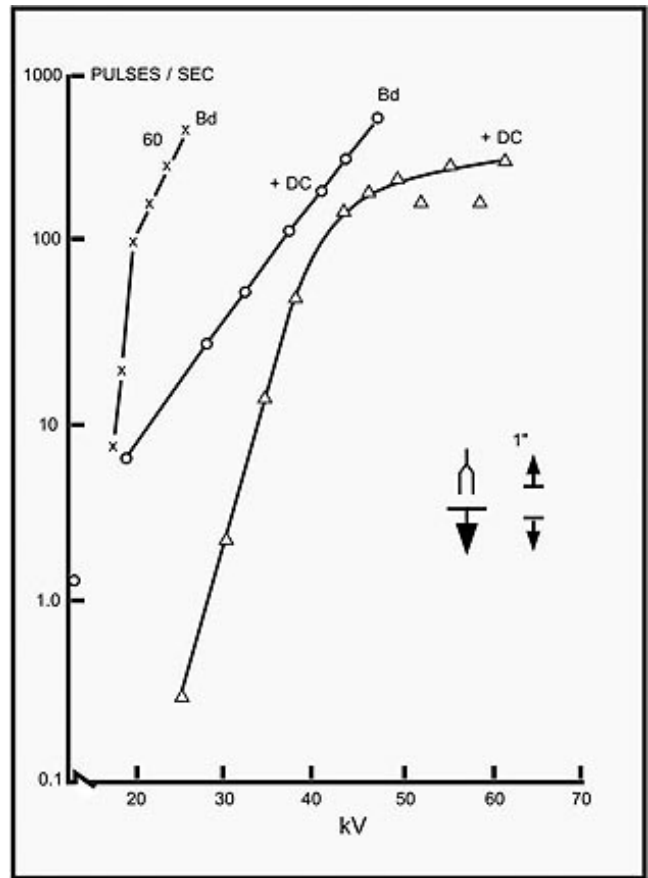

Fig. 7: Discharge repetition rate vs voltage; $12.5 \mathrm{~mm}$ tube to plane spaced $25 \mathrm{~mm}$ (vertical). Iron powder piled on plate. 


\section{Summary and Conclusions}

Particles in oil decrease both its AC and DC breakdown voltage. The breakdown value is dependent upon the particle size and its conductivity. With conducting metal or partially conducting particles (carbon and wet cellulose fibers) the decrease in breakdown strength is significantly greater for direct voltage than for alternating voltage. In this work, the effect of particle concentration or shape has not been adequately defined. It is possible that the observed decrease in breakdown with an increase in particle count may be related more to the presence of large particles at the higher particle concentrations than to the higher concentration itself Possibly both size and concentrations have an effect The use of particle counting in quality control procedures for transformer oil appears to be justified. Lower magnitudes of acceptable particle count are indicated for oil to be used in DC equipment.

Progress has been made in characterizing the shape of particles removed from transformer oil and in recognizing the possibility that small particles may agglomerate to form large particles. These large agglomerates may also break up to smaller particles under both mechanical and electrical stress. The parameters of these competitive processes have not been defined. However, the processes may well be responsible for the apparently contradictory test results that have been obtained in some cases.

With both alternating and direct voltage, particle motion starts at $5 \%$ or less of the breakdown voltage. On the other hand, measurable corona occurs only at voltages above $80 \%$ of the breakdown voltage. With alternating stress and the electrode geometries investigated, steady corona sometimes does not occur before voltage breakdown. Particle concentration in high field region tends first to increase as voltage stress increases; then, at higher stresses or with time, particles tend to be drawn out of the high stress areas at the center of the electrodes.

Since these measurements have all been made in oil alone with relatively small electrode gaps, the pertinence to design. test and service performance is thereby limited. The relatively greater influence of particles and the lower breakdown strength with direct voltage compared with alternating voltage does suggest that conservative design and care in manufacture may be especially important with such DC apparatus. The results suggest, also, that corona in oil (as distinct from gas-filled voids) may be more likely to occur under direct than alternating voltage.

References

1. T Hirose et al, Electrician 78, 656 (1917).

2. J.A. Kok, Electrical Break.doum of Insulating Liquids, Interscience Publishers, Inc., New York (1961).

3. T.J. Gallagher, Simple Dielectric Liquids- Mobility, Conduction and Breakdown, Clarendon Press, Oxford (1975).

4. J.M. Goldschwartz and B.S. Blaise, "Electrical Breakdown of Liquid Helium," Brit J AppL Phys. 17, 1083-89 (1966).

5. Z. Krasucki, "Breakdown of Liquid Dielectrics," Proc. Roy. Soc. A 294, 393-404 (1966).

6. NH. Sharbaugh and P.K. Watson. "Progress in Dielectrics," VoL 4, Hey-wood, London (1962).

7. T.W. Dakin and J. Hughes, Ann. Rept. 1968 Conf. Elec. InsuL Dielec. Phen., p. 68.

8. J. Crawley and L Angerer, "Investigation of the Electrical Breakdown of Transformer Oil in Skowoaski's Cap-Sphere Spark Gap," Proc. TEE VI13NG, pp. 1103-1105 (1966).

9. P.R Flanagan. "The Measurement of Particulate Contaminants in Transformer Oil and Cortelation with $60 \mathrm{~Hz}$ Dielectric Strength," 43rd Annual Internation Conf. of Doble Clients 1976.

10. M. Darveniza, "The Effect of Carbon Particles on the AC Strength of Transformer Oil," Elect Eng. Tr. LE. Aust, Sept. 1969. 
NOTE: Because of lack of space, paragraphs describing voltage breakdown and photographic studies of patterns of particle motion have been omitted. 